\title{
A Novel Multiband Directional Antenna for Wireless Communications
}

\author{
Chun-Xu Mao, Steven Gao, Senior Member, IEEE, Yi Wang, Senior Member, IEEE, Benito Sanz- \\ Izquierdo
}

\begin{abstract}
A novel multiband patch antenna with unidirectional radiation is proposed by integrating resonators in the design. The multiple frequency bands are achieved based on a coupled resonators network. The topology and design methodology are detailed. The patch not only works as the lastorder resonator of the network but also the radiating element. Using this approach, multiple bands can be achieved without changing the shape of the radiation element. In addition, the operation bands can be adjusted by adjusting the coupling between the resonators. In this work, the four bands are designed at 4.6, 5.05, 5.8 and 6.3 GHz as a proof-of-concept. The prototype is fabricated and tested and measured results agree very well with the simulations, showing an excellent performance in terms of impedance matching, radiation patterns, gains and cross polarization discrimination (XPD).
\end{abstract}

Index Terms - Multiband, patch antenna, resonator-based, unidirectional.

\section{INTRODUCTION}

$\mathrm{R}^{\mathrm{s}}$ ECENT RAPID development of wireless communications is promoting various applications such as GSM, CDMA, LTE, Wi-Fi, WLAN. Antenna, as the transmitting/receiving component of the communication systems, are required to have the properties of compact size, high integration and light weight in order to reduce the cost of massive industrial production. To meet the demand for multiband operation, one of useful approaches is to design a broadband antenna to cover all applications/bands. Multiband antenna is believed another feasible method to achieve the goal. In some applications where high gain and pure polarization are required, the antenna with multiband operation and uni-directional radiation is also required.

Many methods for designing the antennas with multiband operation have been investigated [1]-[10]. For compact and portable applications, where the polarization purity is not seriously concerned, the planar inverted-F antenna (PIFA) is often employed to realize multiband operations [1]. In [2], dual/tri-band operations are achieved by adopting the

Manuscript submitted on April 30, 2016; This work is supported by the project "DIFFERENT" funded by EC FP7 (grant no. 6069923). YW is supported by UK EPSRC under Contract EP/M013529/1.

C. Mao, S. Gao and B. Sanz are with School of Engineering and Digital Arts, University of Kent, Canterbury, UK (email: cm688@kent.ac.uk).

Y. Wang is with the Department of Engineering Science, University of Greenwich, UK.

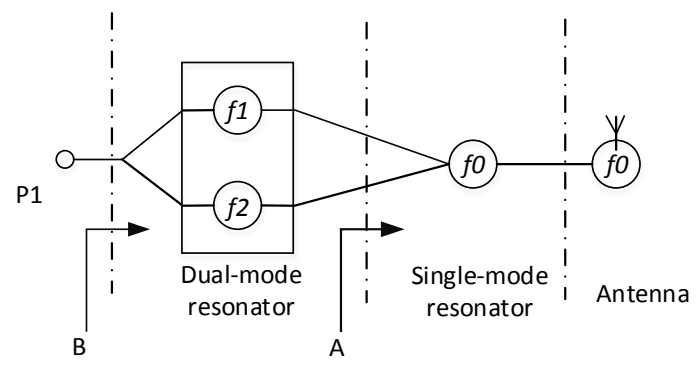

(a)

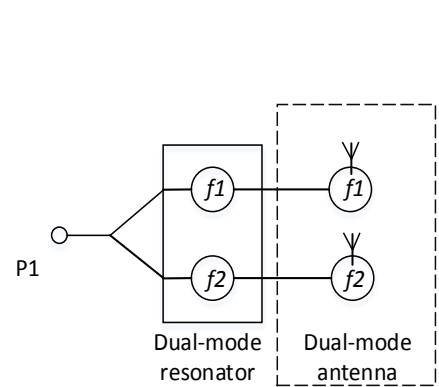

(b)

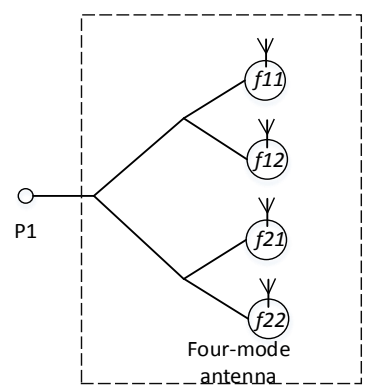

(c)
Fig. 1. The resonator-based topology: (a) topology of the proposed four-band antenna, (b) equivalent topology see from interface A, (c) equivalent topology see from interface $B$.

fundamental and the spurious frequencies of the radiation element. In [3]-[5], multiband antennas with omnidirectional radiation are obtained by loading slots or parasitic stubs on the radiator. For the unidirectional antenna, multiple bands could also be achieved by stacking multiple patches with different sizes, however which leads to high thickness and complexity [6]. Another proposed solution to achieve multiband operation with unidirectional radiation is inserting U-shaped slots in the radiator [7]-[9]. However, such kind of antennas have a poor polarization purity over the different bands, and thus it is not appropriate for using as a transmitter. Besides, those antennas cannot be extended for dual polarizations application due to the asymmetry of the radiating elements.

In this paper, based on a coupled resonator-based circuit model, a novel unidirectional patch antenna with four distinct operation bands is proposed for wireless communication applications. Different from the traditional multiband antennas, the four frequency bands are achieved by integrating some resonators in the antenna design. The four frequency bands can be adjusted by tuning the coupling between the resonators. The prototype is optimized and tested and the measured results agree well with the simulations. 


\section{TOPOLOGY AND EQUiVALENT CIRCUITS}

Fig. 1(a) shows the proposed topology for realizing a fourband antenna. The topology is composed of two $1^{\text {st }}$-order resonator and one $2^{\text {nd }}$-order resonator and thus $4^{\text {th }}$-order resonant characteristics can be achieved. The circles represent the resonators and the lines represent the coupling. It should be noted that the patch, as the radiating element, also serves as the last-order resonator of the network.

The design concept is detailed as follows. First, the dimension of the patch is chosen with its resonant frequency $\left(f_{0}\right)$ located around the central frequency of the four designate operation bands. Then, the patch is coupled to a single-mode resonator which has the same resonant frequency $\left(f_{0}\right)$. By adjusting the coupling between them, the resonator-fed patch can split into two resonant frequencies, denoted as $f_{1}$ and $f_{2}$. Seeing from the interface A in Fig. 1(a), the coupled resonator-patch is equivalent to a dual-band antenna $\left(f_{1}, f_{2}\right)$ and thus the topology is converted to the topology in Fig. 1(b). The coupled resonator-patch is then coupled to a dual-mode resonator which also has the resonant frequencies of $f_{1}$ and $f_{2}$. The dual-mode resonator and the dual-band antenna are tuned at each band respectively, generating two groups of resonant frequencies: $f_{11}, f_{12}$ and $f_{21}, f_{22}$. These four resonant frequencies will result in four distinct frequency bands. Seeing from the port (interface B), the topology in Fig. 1(a) can be equivalent to a four-band antenna, as shown in Fig. 1(c). The topology study provide a new circuit method to achieve multiband antenna using a single-mode antenna.

\section{ANTENNA DESIGN}

\section{A. Configuration}

Based on the topology in Fig. 1(a), a multiband patch antenna is conceived and the configuration is shown in Fig. 2. The antenna is composed of two stacked substrates and a foam of $1 \mathrm{~mm}$ between them. The radiating element (patch) is printed on the top layer of the upper substrate whereas the resonators and the feeding network are printed on the bottom layer of the lower substrate. Both of them share the same ground plane in the middle. A slot is etched in the ground for coupling the electromagnetic energy from the resonator in the bottom layer to the patch on the top layer. The hairpin is then coupled to a dual-mode stub-loaded resonator (SLR). Rogers 4003 substrate with relative permittivity of 3.55 and loss tangent of 0.0027 is used in this design.

Since the TM10 mode of the patch is used in the design, the dimension of the patch is about half-wavelength. The dimensions of the hairpin and the SLR can be approximately calculated using the following expressions [10-12],

$$
\begin{aligned}
& 2 L 1+L 2 \approx \frac{c}{2 f_{0} \sqrt{\varepsilon_{\text {eff } 2}}} \approx L p \\
& 2 L 1+L 3 \approx \frac{c}{2 f_{1} \sqrt{\varepsilon_{\text {eff } 2}}} \\
& L 1+\frac{L 3}{2}+L 4 \approx \frac{c}{2 f_{2} \sqrt{\varepsilon_{\text {eff } 2}}}
\end{aligned}
$$

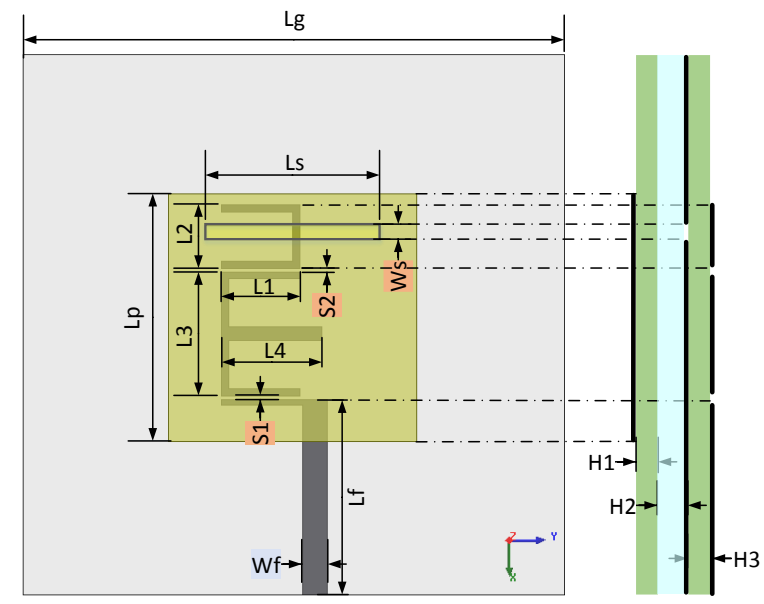

(a)

(b)

Fig. 2. Configuration of the proposed four-band patch antenna: (a) top view, (b) side view.

TABLE I

PARAMETERS OF THE ANTENNA PROPOSED: (MM)

\begin{tabular}{cccccccc}
\hline \hline $\mathrm{Lg}$ & $\mathrm{Lp}$ & $\mathrm{Ls}$ & $\mathrm{Lf}$ & $\mathrm{L} 1$ & $\mathrm{~L} 2$ & $\mathrm{~L} 3$ & $\mathrm{~L} 4$ \\
50 & 17.6 & 12.4 & 18.7 & 5.5 & 4.5 & 8.8 & 7 \\
\hline $\mathrm{Wf}$ & $\mathrm{Ws}$ & $\mathrm{S} 1$ & $\mathrm{~S} 2$ & $\mathrm{H} 1$ & $\mathrm{H} 2$ & $\mathrm{H} 3$ & \\
1.8 & 0.9 & 0.25 & 0.25 & 0.813 & 1 & 0.813 & \\
\hline \hline
\end{tabular}

where, $\varepsilon_{\text {eff }}$ is the effective relative permitivity, $c$ is the speed of light in free space. $f_{1}$ and $f_{2}$ are the odd- and even-mode resonant frequencies of the SLR. The design and optimization were performed using HFSS 15, and the optimized parameters are presented in Table I.

\section{B. Design methodology}

To achieve a four-band patch antenna, a dual-band antenna is first designed. According to the filter design theory, $2^{\text {nd }}-$ order resonant characteristics can be achieved when the patch is synchronically tuned with a single-mode resonator, resulting in dual-band operation. The two frequency bands can be adjusted to the designate locations by tuning the coupling intensity between them. Fig. 3 presents the simulated $S_{11}$ and the configuration of the dual-band patch antenna. The hairpin and the patch are designed to resonate at $5.4 \mathrm{GHz}$. As can be observed, two bands at 4.8 and $5.85 \mathrm{GHz}$ are achieved.

In this design, coupling plays an important role in adjusting the location of the two resonant modes. Here, mixed electric and magnetic coupling is used between the resonators. The coupling strength can be controlled by adjusting the dimension of the coupling slot in the ground. Fig. 4 shows the results of extracted ratio of $f_{2}$ and $f_{1}$ with different lengths of the coupling slot, as the $L s$ indicated in Fig. 2. As can be observed, the ratio of $f_{2}$ and $f_{1}$ increases from 1.05 to 1.32 when the length of the slot increases from 7 to $16 \mathrm{~mm}$.

In order to obtain four frequency bands, the dual-band hairpin-patch is coupled to a SLR. The two operation bands and the two modes of the resonator are synchronically tuned. As a result, the two frequency bands $\left(f_{1}\right.$ and $\left.f_{2}\right)$ are split into four resonant modes, denoted as $f_{11}, f_{12}$ and $f_{21}, f_{22}$ and four operation bands are obtained. The locations of the four bands can be controlled by adjusting the coupling strength between the SLR and the hairpin. Fig. 5 shows the extracted ratios of 


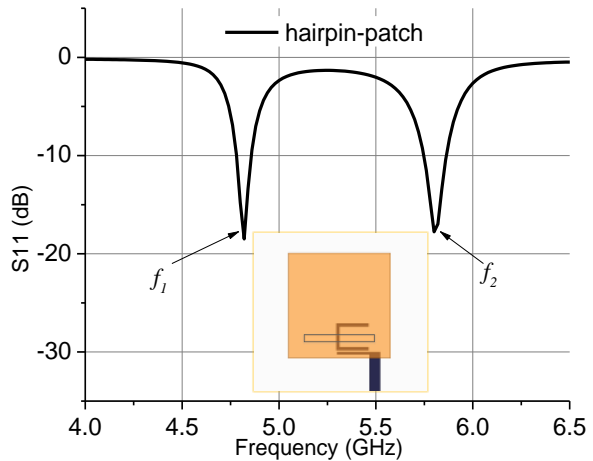

Fig. 3. The simulated $S_{11}$ and the configuration of the dual-band antenna.

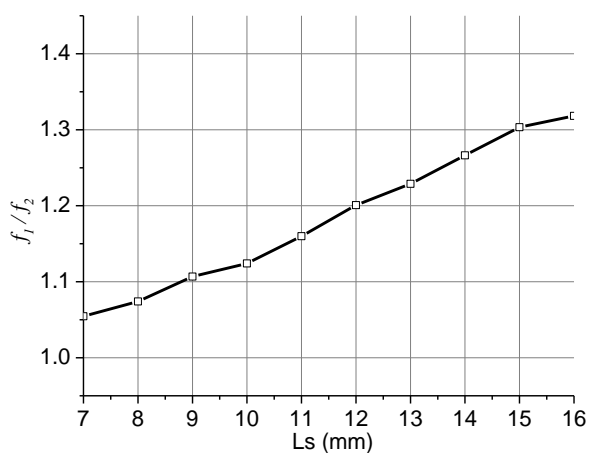

Fig. 4. The ratio of the two bands with different lengths of the slot.

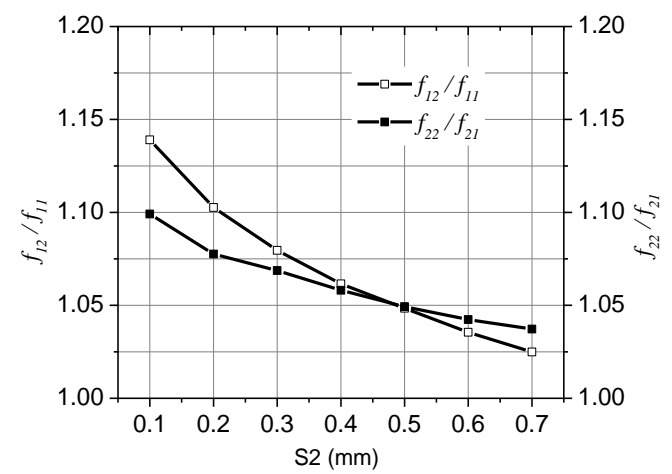

Fig. 5. The frequency ratios of $f_{12} / f_{11}$ and $f_{22} / f_{21}$ with different $S 2$.

the first two frequency bands $\left(f_{12} / f_{11}\right)$ and the last two frequency bands $\left(f_{22} / f_{21}\right)$ with different $S 2$. When $S 2$ increases from 0.1 to $0.7 \mathrm{~mm}$, the ratios of $f_{12}$ and $f_{11}\left(f_{22}\right.$ and $\left.f_{21}\right)$ are decreases correspondingly.

Fig. 6 shows the simulated $S_{11}$ of the proposed four-band patch antenna with different lengths of the slot, Ls. It is observed the first and the second bands move to lower frequency band simultaneously as the $L s$ increases from 12 to $13.5 \mathrm{~mm}$ while the third and the fourth bands maintain unchanged. Fig. 7 shows the $S_{11}$ of the antenna with different spaces between the SLR and hairpin, $S 2$. As can be seen, when the $S 2$ reduces from $0.5 \mathrm{~mm}$ to $0.2 \mathrm{~mm}$, the first and the third bands moves to lower band whereas the second and the fourth bands moves to higher band.

\section{Current Distribution}

As a proof-of-concept, the four bands of the antenna are designed at 4.6, 5.05, 5.8 and $6.3 \mathrm{GHz}$. Fig. 8 shows the current distribution of the antenna at the four frequencies. It is

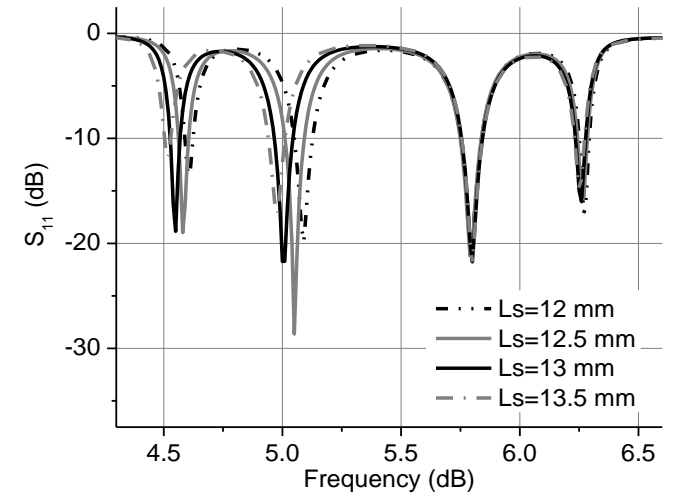

Fig. 6. The $S_{11}$ of the four-band antenna with different $L s$.

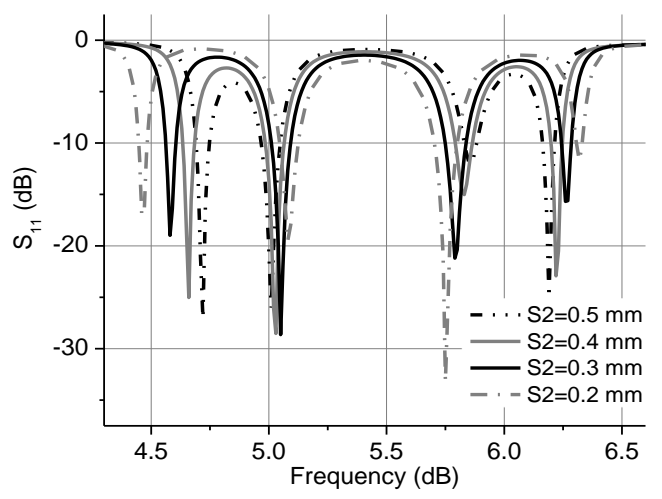

Fig. 7. The $S_{11}$ of the four-band antenna with different $S 2$.

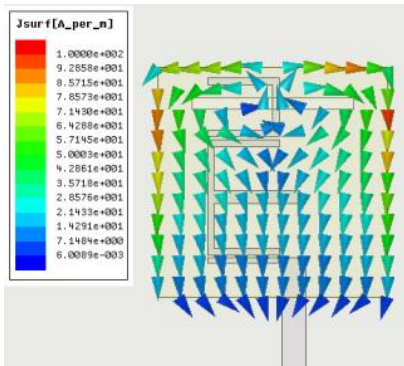

(a)

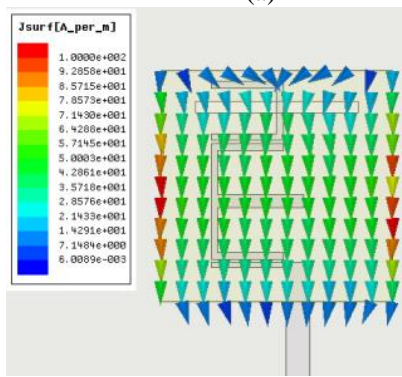

(c)

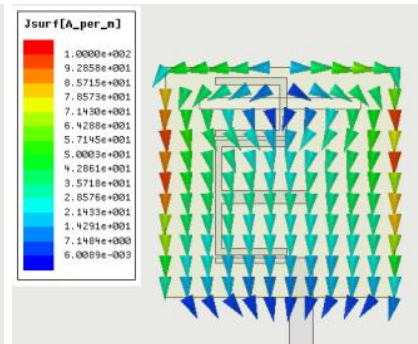

(b)

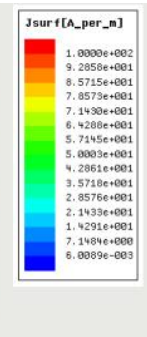

(d)
Fig. 8. The current distribution at the four operating frequencies: (a) 4.6 $\mathrm{GHz}$, (b) $5.05 \mathrm{GHz}$, (c) $5.8 \mathrm{GHz}$ and (d) $6.3 \mathrm{GHz}$.

observed that the antenna has a similar current distribution characteristics at the four bands and therefore the antenna has consistent radiation patterns and high polarization purity at the four bands.

\section{RESULTS AND DISCUSSION}

Fig. 9 shows the simulated and measured $S_{11}$ of the proposed four-band patch antenna. As can be observed, the 


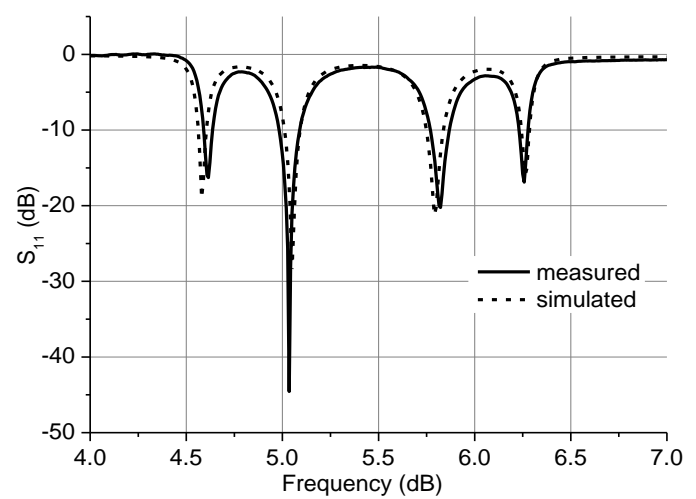

Fig. 9. The simulated and measured $S_{11}$ of the proposed antenna.

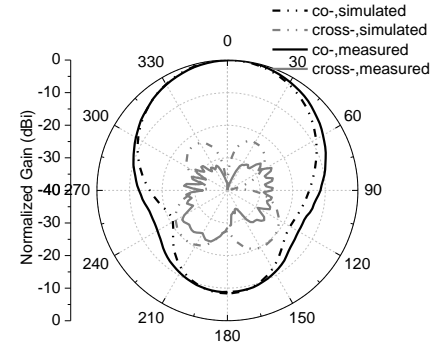

(a)

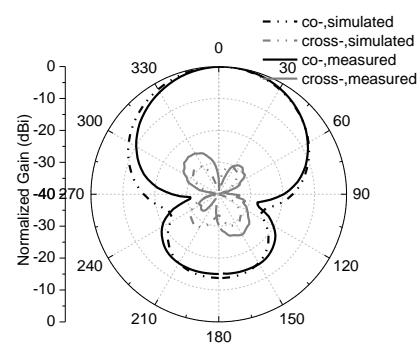

(c)

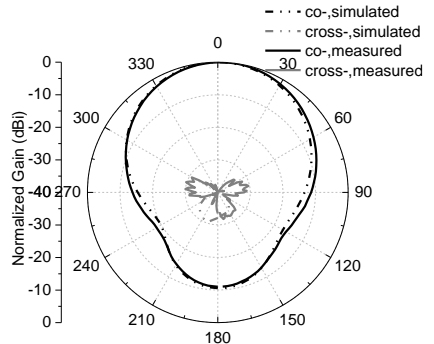

(b)

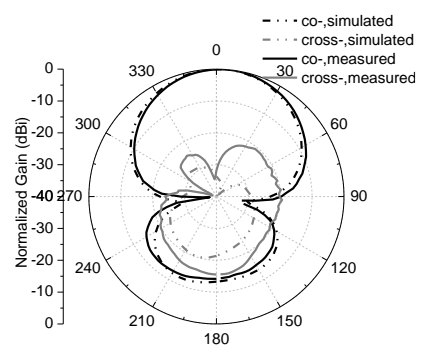

(d)
Fig. 10. The simulated and measured normalized E-plane radiation patterns at the four frequencies: (a) $4.6 \mathrm{GHz}$, (b) $5.05 \mathrm{GHz}$, (d) $5.8 \mathrm{GHz}$ and (d) 6.3 $\mathrm{GHz}$.

measured result agrees very well with the simulation, showing four operation bands at around 4.6, 5.05, 5.8 and $6.3 \mathrm{GHz}$, respectively. The measured bandwidths of the four bands are $1.2 \%, 2 \%, 1.9 \%$ and $0.9 \%$. The minor discrepancy (frequency shifts) is mainly attributed to the fabrication errors.

Figs. 10(a)-(d) show the simulated and measured radiation patterns in E-plane at 4.6, 5.05, 5.8 and $6.3 \mathrm{GHz}$, respectively. As can be seen, measured and simulated results agree well with each other, showing similar uni-directional radiation patterns and very low cross polarization level. At the four frequencies, the radiation pattern achieve a XPD lower than $30 \mathrm{~dB}$ in $\theta=0^{0}$ and the front-back ratio is better than $10 \mathrm{~dB}$. Fig. 11 shows the simulated and measured antenna gain from 4.0 to $7.0 \mathrm{GHz}$. As can be observed, the proposed antenna has four peak gain points at 4.6, 5.05, 5.8 and $6.3 \mathrm{GHz}$, respectively.

\section{CONCLUSION}

A novel multiband uni-directional antenna based on a coupled resonators-based network is proposed in this paper. By conceiving a resonant network, multiple frequency bands

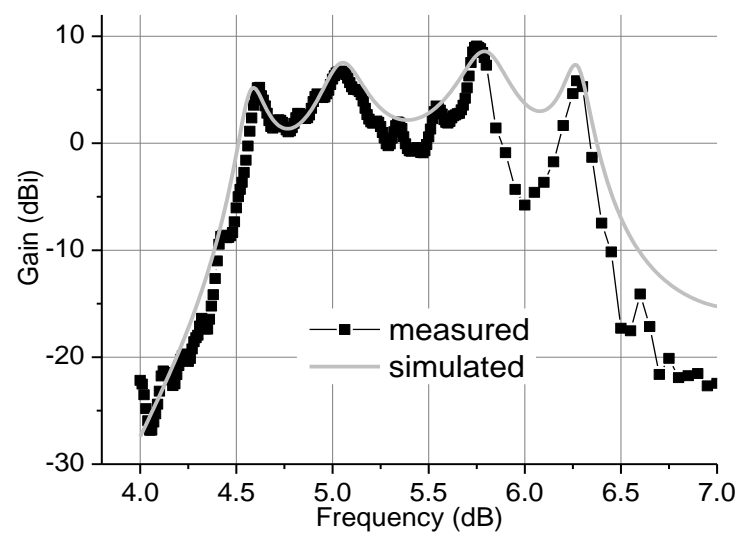

Fig. 11. The simulated and measured antenna gains.

can be achieved by using a single-mode patch antenna. The methodology and design process are detailed with the study of the parameters. The four frequency bands can be adjusted by changing the coupling strength between the resonators. In addition, the operation bands can be adjusted without changing the shape of the antenna, providing a flexible method in antenna design. Simulated and measured results show that the proposed antenna has very good impedance matching and radiation performance at the four distinct frequency bands. This paper provides a novel method to achieve multiband antenna based on circuit methods. The antenna can also be further developed to dual-polarized antennas and high gain array antennas.

\section{REFERENCES}

[1] L. Pazin, N. Telzhengsky and Y. Leviatan, "Multiband flat-plate inverted-F antenna for Wi-Fi/WiMax operation," IEEE Antennas and Propag., vol. 7, pp. 197-200, 2008.

[2] S. W. Chen, D. Y. Wang and W. H. Tu, "Dual-band/tri-band/broadband CPW-fed stepped-impedance slot dipole antennas," IEEE Trans. Antennas and Propag., vol. 62, no. 1, pp. 485-490, Jan. 2014.

[3] K. Seol, J. Jung and J. Choi, "Multiband monopole antenna with inverted U-shaped parasitic plane," Electronics Lett., vol. 42, no. 15, pp. 844-845, 2006.

[4] Y. F. Cao, S. W. Cheung and T. I. Yuk, "A multiband slot antenna for GPS/WiMAX/WLAN systems," IEEE Trans. Antennas and Propag., vol.63, no. 3, pp. 952-958, Mar. 2015.

[5] H. Li, Y. Zhou, X. Mou, Z. Ji, H. Yu and L. Wang, "Miniature fourband CPW-fed antenna for RFID/WiMAX/WLAN applications," IEEE Antennas and Propag., vol. 13, pp. 1684-1688, 2014.

[6] J. Anguera, G. Font, C. Puente, C. Borja, and J. Soler, "Multi-frequency microstrip patch antenna using multiple stacked elements," IEEE Microw. Wireless Compon. Lett., vol. 13, no. 3, pp. 123-124, 2003.

[7] W. C. Mok, S. H. Wong, K. M. Luk and K. F. Lee, "Single-layer singlepatch dual-band and triple-band patch antenna," IEEE Trans. Antennas and Propag., vol. 61, no. 8, pp. 4341-4344, Aug. 2013.

[8] K. Jhamb, L. Li, K. Rambabu, "Frequency adjustable microstrip annular ring patch antenna with multiband characteristics," IET Microw. Antennas Propag., vol. 5, no. 12, pp. 1471-1478, 2011.

[9] S. Liu, S. S. Qi, W. Wu, D. G. Fang, "Single-layer single-patch fourband asymmetrical U-slot patch antenna," IEEE Trans. Antennas and Propag., vol. 62, no. 9, pp. 4895-4899, Sep. 2014.

[10] J. S. Hong and M. J. Lancaster, Microwave Filter for RF/Microwave Application. New York: Wiley, 2001.

[11] C. X. Mao, S. Gao, Y. Wang, F. Qin and Q. X. Chu, "Multi-mode resonator-fed dual polarized antenna array with enhanced bandwidth and selectivity," IEEE Trans. Antennas and Propag., vol. 63, no. 12, pp. 5492-5499, Dec. 2015.

[12] X. Y. Zhang, J. X. Chen, Q. Xue and S. M. Li, "Dual-band Bandpass Filters Using Stub-Loaded Resonators," Micro. and Wireless Components Lett., vol. 17, No. 8, pp. 583-585, Aug. 2007. 